\title{
The influence of print exposure on the body-object interaction effect in visual word recognition
}

\author{
Dana Hansen ${ }^{1}$, Paul D. Siakaluk ${ }^{1 *}$ and Penny M. Pexman ${ }^{2}$ \\ ' Department of Psychology, University of Northern British Columbia, Prince George, BC, Canada \\ ${ }^{2}$ Department of Psychology, University of Calgary, Calgary, AB, Canada
}

\author{
Edited by: \\ Melvin Yap, National University \\ of Singapore, Singapore \\ Reviewed by: \\ Chi-Shing Tse, The Chinese \\ University of Hong Kong, \\ Hong Kong \\ Mark Yates, University of South \\ Alabama, USA

\section{*Correspondence:} \\ Paul D. Siakaluk, Department of \\ Psychology, University of Northern \\ British Columbia, Prince George, \\ BC V2N 4Z9, Canada. \\ e-mail:siakaluk@unbc.ca
}

We examined the influence of print exposure on the body-object interaction (BOI) effect in visual word recognition. High print exposure readers and low print exposure readers either made semantic categorizations ("Is the word easily imageable?"; Experiment 1) or phonological lexical decisions ("Does the item sound like a real English word?"; Experiment 2). The results from Experiment 1 showed that there was a larger $\mathrm{BO}$ effect for the low print exposure readers than for the high print exposure readers in semantic categorization, though an effect was observed for both print exposure groups. However, the results from Experiment 2 showed that the BOI effect was observed only for the high print exposure readers in phonological lexical decision. The results of the present study suggest that print exposure does influence the $\mathrm{BOI}$ effect, and that this influence varies as a function of task demands.

Keywords: embodied cognition, perceptual symbol systems, motor simulation, print exposure, lexical conceptual processing
The body-object interaction (BOI) variable measures perceptions of the ease with which a human body can physically interact with a word's referent (Siakaluk et al., 2008a). As such, high BOI words (e.g., mask) refer to objects with which a human body can easily interact, whereas low BOI words (e.g., ship) refer to objects with which a human body cannot easily interact. In recent research the effects of BOI have been examined in a variety of visual word and object recognition tasks.

Siakaluk et al. (2008a) examined the effects of BOI in a lexical decision task; they manipulated BOI while controlling for the effects of numerous confound variables known to influence visual word recognition performance including, importantly, imageability and concreteness (i.e., their high BOI words and low BOI words were equally imageable and concrete). They reported a facilitatory effect of BOI, such that high BOI words were responded to more rapidly than low BOI words. Since this initial study, facilitatory effects of BOI have been reported for lexical decision in two studies using much larger sets of monosyllabic words (Tillotson et al., 2008; Siakaluk et al., 2011, Experiment 3) and in two studies using large sets of multisyllabic words (Bennett et al., 2011; Yap et al., 2012b).

The effects of BOI have also been examined in tasks in which responses are based primarily on phonological processing. Siakaluk et al. (2008a) used a phonological lexical decision task in which words, pseudohomophones (e.g., brane), and pronounceable non-words (e.g., frane) were used and the decision category was, "Does the item sound like a real English word?" In this task, "yes" responses were made to the words and pseudohomophones, whereas "no" responses were made to the pronounceable nonwords. In addition, the effects of BOI have been examined in word naming (Bennett et al., 2011; Yap et al., 2012b) and picture naming tasks (Bennett et al.). As was the case for lexical decision, a facilitatory BOI effect was reported for each of these tasks.

Lastly, the effects of BOI have been examined in the semantic categorization task. Siakaluk et al. (2008b) used three different versions of this task. In Experiments $1 \mathrm{~A}$ and $1 \mathrm{~B}$, the same set of high BOI words, low BOI words, and less imageable word foils were used. For Experiment 1A the decision category was, "Does the word refer to something that is easily imageable?", whereas for Experiment $1 \mathrm{~B}$ the decision category was, "Does the word refer to something that is not easily imageable?" Thus, in the first experiment the experimental items required a "yes" response, whereas in the second experiment they required a "no" response. A facilitatory BOI effect was reported for both experiments. In Experiment 2, they conducted what they called a semantic lexical decision task. In this task, the high BOI words, low BOI words, and less imageable word foils were intermixed with pseudohomophones. There were two decisions that were required: first to decide if the item was a word or not, and second, if the item was a word, to decide if it was easily imageable or not. Again, a facilitatory BOI effect was reported, and, interestingly, it was significantly larger than that observed in Experiment 1A (in which only one decision needed to be made). Since this initial study, a facilitatory BOI effect has been reported in semantic categorization tasks (using the same decision category as used in Siakaluk et al., 2008b) in which verbal responses were used (Wellsby et al., 2011) and in which multisyllabic words were used (Bennett et al., 2011; Yap et al., 2012b, who used a "Does the word refer to something that is concrete?" decision category).

More recently, Tousignant and Pexman (2012) examined the effects of BOI in four versions of a semantic categorization task. The same set of high BOI words, low BOI words, and action words 
were used in each version, but the framing of the decision category varied between versions. The four decision categories (and sets of instructions) were: "Is it an entity?" (press the left button for entities and the right button for non-entities); "Is it an entity or an action?" (press the left button for entities and the right button for actions); "Is it an action or an entity?" (press the left button for actions and the right button for entities); and "Is it an action?" (press the left button for actions and the right button for non-actions). Tousignant and Pexman reported a facilitatory BOI effect in all three versions in which the instructions included entity words as part of the decision category, but not in the version in which the instructions did not include entity words as part of the decision category. They proposed that BOI information is used under conditions in which object information is made salient, such as when participants are expecting to see entity words (which are concrete nouns that refer to concepts with which human bodies can physically interact), but not for action words (which are verbs that refer to concepts with which human bodies cannot physically interact).

There are two frameworks that, when combined, have been used to provide an explanatory account for facilitatory effects of BOI. The first is an influential embodied cognition framework known as perceptual symbol systems (Barsalou, 1999, 2008). Embodied cognition more generally is the theoretical perspective that much of human cognition is acquired through (or grounded in) sensorimotor experience with the environment (Clark, 1997; Lakoff and Johnson, 1999; Wilson, 2002; Pecher and Zwaan, 2005). There are two key assumptions of the perceptual symbol systems framework that are relevant for an explanatory account of facilitatory BOI effects. The first assumption is that lexical conceptual knowledge is multimodal. That is, there are multiple neural systems involved in the acquisition and retrieval of lexical conceptual knowledge. Among these are neural systems dedicated to processing sensory knowledge (e.g., visual, auditory), emotional knowledge (e.g., fear, excitement), introspective knowledge (e.g., association, thought), and, most relevant in accounting for the facilitatory effects of BOI, motor, kinesthetic, and proprioceptive knowledge (e.g., physically interacting with objects, internal feedback from muscles and joints). The second assumption is that retrieving lexical conceptual knowledge from memory involves the process of simulation. Simulation refers to the partial re-enactment of the states of the various neural systems that were involved at the time of encoding. Importantly, these assumptions were supported by a recent fMRI study examining the effects of BOI in a semantic categorization task that used the imageability decision category (Hargreaves et al., 2012a). Hargreaves and colleagues reported that in addition to a large facilitatory behavioral effect of BOI, greater activation was observed for high BOI words than for low BOI words in the left inferior parietal lobule (i.e., the supramarginal gyrus, BA $40)$, which is a brain region associated with kinesthetic memory (Grèzes and Decety, 2001; Péran et al., 2010).

We have previously extended the perceptual symbol systems framework to provide an explanatory account for facilitatory effects of BOI in the following way (e.g., Siakaluk et al., 2008a,b). As noted, high BOI words refer to objects that human bodies can easily physically interact with, whereas low BOI words refer to objects that human bodies cannot easily physically interact with. Thus, the former types of words will develop and eventually elicit richer motor, kinesthetic, and proprioceptive representations than will the latter types of words. Stated another way, using the terminology of perceptual symbol systems, high BOI words will develop and eventually elicit richer motor, kinesthetic, and proprioceptive simulations (we will hereafter simply refer to these different types of simulations as motor simulations) than will low BOI words.

The second framework that is relevant to an explanatory account for facilitatory effects of BOI is the semantic feedback framework (Hino and Lupker, 1996; Pexman and Lupker, 1999; Pecher, 2001; Hino et al., 2002). This framework has three important assumptions and two mechanisms by which facilitatory effects of BOI may arise. The first assumption is that different word characteristics are processed in different, dedicated sets of units. That is, orthographic knowledge is processed within orthographic units, phonological knowledge is processed within phonological units, and semantic knowledge is processed within semantic units. The second assumption is that these three sets of units are interconnected such that the processing of one set of units may influence the processing of another set of units. The third assumption is that the impact of the processing of one set of units on another set of units is dependent on the nature of the connections between the two sets of units. One mechanism is involved in tasks in which responses are based primarily on semantic processing (e.g., semantic categorization). A facilitatory BOI effect arises in these tasks because high BOI words elicit richer semantic activation (i.e., richer motor simulations) within the semantic units that leads to faster settling on a semantic representation and hence faster semantic categorization latencies. The other mechanism is involved in tasks in which responses are based primarily on either orthographic processing (e.g., lexical decision) or phonological processing (e.g., phonological lexical decision). A facilitatory BOI effect arises in these tasks because high BOI words elicit greater semantic activation (i.e., richer motor simulations) within the semantic units, which then sends stronger semantic feedback to the orthographic units and to the phonological units, leading to faster settling on an orthographic representation or phonological representation and hence faster lexical decision latencies and phonological lexical decision latencies, respectively. Thus, facilitatory BOI effects have been explained by this combination of the perceptual symbol systems framework for embodied semantic knowledge and the semantic feedback framework for the visual word recognition system.

Zwaan (2008) suggested several avenues of future research for those interested in studying embodiment effects in language, one of which was "to examine more closely the role of prior experience ... in language comprehension" (p. 172). Although perhaps not exactly what Zwaan had in mind when he gave this recommendation, we were interested in a related idea: examining whether prior reading experience (i.e., print exposure) would modulate the facilitatory effects of BOI in visual word recognition, using semantic categorization and phonological lexical decision tasks. Before presenting in more detail the purpose of the present study, we will discuss the lexical integrity hypothesis 
(Yap et al., 2009), which is integral to the predictions we make below regarding the influence of print exposure on facilitatory effects of BOI.

Yap et al. (2009) developed the lexical integrity hypothesis (see also the lexical quality hypothesis, e.g., Perfetti, 1992; Perfetti and Hart, 2002; Andrews and Bond, 2009) to account for several findings regarding the influence of semantics (and lexical variables such as print frequency) in the literature. For example, in their study, Yap et al. examined the joint influence of semantic priming and word frequency in lexical decision. Their primary findings were that these two variables have interactive effects (i.e., larger effects of semantic priming for low-frequency words than for high-frequency words) for readers with less vocabulary knowledge, but have additive effects (i.e., similar effects of semantic priming for low-frequency words and for high-frequency words) for readers with more vocabulary knowledge. Yap et al.'s notion of lexical integrity accounts for these findings in the following way. Readers with more vocabulary knowledge develop higher integrity orthographic representations that are closer to recognition threshold, whereas readers with less vocabulary knowledge have lower integrity orthographic representations that are further removed from recognition threshold. Importantly, the difference in lexical integrity between the two types of readers is likely to be larger for lower frequency words. Yap et al. state it this way, "a medium-frequency word for a high-lexical-integrity individual is likely to be a low-frequency word for a low-lexical-integrity individual" (p. 306). In general, because orthographic representations further away from recognition threshold require more lexical conceptual processing before lexical decisions can be made, they would benefit more from, say, semantic priming. Thus, Yap et al. predicted that, "one might actually expect individuals with lower integrity representations to show a larger influence of semantic context than those with higher integrity representations" (p. 306; emphasis in original). This reasoning can also account for the recent demonstration that non-expert Scrabble players showed larger effects of concreteness in lexical decision than did competitive Scrabble players, who have considerable lexical knowledge (Hargreaves et al., 2012b).

We propose to extend the lexical integrity hypothesis in the following ways. First, we assume that readers with more print exposure, in addition to developing higher integrity orthographic representations, also develop higher integrity semantic representations and higher integrity phonological representations. Second, as noted above in our discussion of the semantic feedback framework, the recognition threshold that needs to be exceeded for responding depends on task demands. That is, semantic categorizations are based primarily on semantic processing, and responses are made available when semantic representations exceed recognition threshold; lexical decisions are based primarily on orthographic processing, and responses are made available when orthographic representations exceed recognition threshold; and phonological lexical decisions are based primarily on phonological processing, and responses are made available when phonological representations exceed recognition threshold. Recall that, according to the semantic feedback framework, in the latter two cases, orthographic processing and phonological processing may be influenced by semantic feedback.

\section{THE PRESENT STUDY}

The purpose of the present study was to examine the influence of print exposure on facilitatory effects of BOI in semantic categorization and phonological lexical decision. To measure print exposure we used the Canadian version of the Author Recognition Test (ART) (Chateau and Jared, 2000). This version is based on the ART originally developed by Stanovich and West (1989). Stanovich and West developed the ART to overcome social-desirability effects in the assessment of print exposure. The ART consists of a list of names, some of which are popular writers of books, magazine articles, and/or newspaper columns (e.g., Margaret Atwood) and some of which are not (e.g., Anne Cunningham). Participants are instructed to only put a check mark next to the names of the individuals whom they know to be writers, and guessing is discouraged because incorrect responses are penalized. The ART has received extensive validation, such that ART scores are associated with early reading ability (Cunningham and Stanovich, 1997), reading experience (Cunningham and Stanovich, 1990), and, most importantly for the present study, vocabulary knowledge (West et al., 1993; Lee et al., 1997), such that higher ART scores predict greater vocabulary knowledge. Thus, the benefits of using the ART in measuring print exposure are that it avoids concerns of social-desirability effects, it is reliably associated with many characteristics of reading experience and ability, and it is relatively cheap to use in terms of required resources (e.g., it is freely available, takes only a few minutes for participants to complete, and is easy to score).

As noted, the present research is concerned with examining the effects of print exposure on facilitatory effects of BOI in semantic categorization and phonological lexical decision. We will first address the experimental procedure, and the predictions and results of the influence of print exposure on BOI in semantic categorization. We will postpone addressing these issues regarding the phonological lexical decision task until after our discussion of the semantic categorization task.

As described below, a high print exposure group and a low print exposure group performed a semantic categorization task in which the decision category was, "Does the word refer to something that is easily imageable?" We used a go/no-go procedure (in which participants responded only to the experimental items), rather than a yes/no procedure (in which participants would respond to both the experimental items and the foil items). Siakaluk et al. (2003) proposed that the go/no-go procedure should elicit more extensive semantic processing than the yes/no procedure using the following reasoning: because overt responses under go/no-go conditions are made only to the experimental items, this may lead participants to adopt a stricter decision criterion to ensure correct responses are made, which would allow for more extensive semantic processing to occur. Further, Siakaluk et al. (2003) predicted that there should be longer response latencies and lower error rates using the go/no-go procedure than the yes/no procedure. These two predictions were supported in their study, as well as in Siakaluk et al. (2007). Most importantly, in both these studies, semantic richness effects were more robust using the go/no-go procedure.

Based upon the lexical integrity hypothesis (Yap et al., 2009), we made the following two predictions. First, there should be a 
main effect of print exposure such that high print exposure readers should exhibit faster response latencies than low print exposure readers. Second, the facilitatory BOI effect should be smaller for high print exposure readers than for low print exposure readers. We made these two predictions based on the reasoning that: (1) high print exposure readers should have higher integrity semantic representations that should be closer to recognition threshold and should thus benefit less from the richer motor simulations evoked by high BOI words; whereas (2) low print exposure readers should have lower integrity semantic representations that are further from recognition threshold and should thus benefit more from the richer motor simulations evoked by high BOI words.

\section{EXPERIMENT 1 \\ METHODS \\ Participants}

Ninety-two undergraduate students from the University of Northern British Columbia participated in the experiment for bonus course credit. All participants were native English-speakers and reported normal or corrected-to-normal vision.

Participants were administered a Canadian version of the ART (Chateau and Jared, 2000) after they completed the semantic categorization task (described below) ${ }^{1}$. For the data analyses, two groups of participants were created using a quartile split of the ART scores. As such, 23 participants were assigned to the high print exposure group (with a mean ART score of 17.0 and a range of 11-33) and 23 participants were assigned to the low print exposure group (with a mean ART score of 3.9 and a range of 3-5).

\section{Stimuli}

The experimental stimuli consisted of the 24 high BOI words (e.g., mask) and the 24 low BOI words (e.g., ship) used in Siakaluk et al. (2008b). The two sets of words were matched for print length, objective print frequency (using HAL log frequency norms from the English Lexicon Project database; Balota et al., 2007), subjective frequency, orthographic and phonological neighborhood size, phonological feedback inconsistency, contextual dispersion, semantic distance, number of features, senses, and associates, and importantly, concreteness and imageability (all $p$ 's $>0.15$ ). In addition, each word only had one entry in the ITP Nelson Canadian Dictionary, (1997) and all had noun definitions listed first. The descriptive statistics for the experimental

\footnotetext{
${ }^{1}$ The version of the Canadian ART we administered had 58 names of authors and 57 names of non-authors, for a total of 115 names.
}

stimuli are listed in Table 1. The 48 less imageable noun foils (e.g., fate) used in Siakaluk et al. (2008b) were also used, and they had a mean imageability rating of 2.6 and a mean printed frequency of 18.9. All the stimuli are listed in the Appendix.

\section{Apparatus and procedure}

The stimuli were presented on a color VGA monitor driven by a Pentium-class microcomputer running DirectRT software (http://www.empirisoft.com/DirectRT.aspx). A trial was initiated by a fixation marker appearing in the center of the computer display. The fixation marker was presented for $1 \mathrm{~s}$ and was then replaced by a word. The participants' task was to decide whether the words were easily imageable or not. Participants were instructed to press the "?" key on the computer keyboard when the imageable words were presented, and to make no response when the less imageable words were presented. For trials in which no response was made, stimulus items remained on the computer display for $2.5 \mathrm{~s}$, and were then removed and replaced by the fixation marker. Participants were further instructed to make their responses as quickly but as accurately as possible. Response latencies were measured to the nearest ms. The order in which the stimuli were presented was separately randomized for each participant. The intertrial interval was $2 \mathrm{~s}$.

Before beginning the experiment, each participant completed 20 practice trials that consisted of 10 imageable words and 10 less imageable words. All the practice stimuli were similar in printed frequency to the stimuli used in the experiment.

\section{RESULTS}

Data for the low BOI word tribe were excluded from the analyses because the error rate for this item was $52.2 \%$. The removal of this item did not affect the matching for the two sets of BOI words for any of the control variables listed above (all $p^{\prime}$ s $>0.20$ ).

Outliers were identified in the following manner. First, response latencies faster than $250 \mathrm{~ms}$ or slower than $2000 \mathrm{~ms}$ were considered outliers. Second, for each participant, response latencies greater than 2.5 SDs from the cell mean of each condition were considered outliers. Using this procedure, a total of 69 observations $(3.2 \%$ of the data) were removed from the data-set.

Response latencies for correct responses and error percentages were analyzed using a 2 (print exposure: high, low) $\times 2$ (BOI: high, low) mixed-model analysis of variance (ANOVA). Both subject $\left(F_{1}\right)$ and item $\left(F_{2}\right)$ analyses were conducted. In the subject analyses, print exposure was a between-subjects variable and BOI was a within-subjects variable. In the item analyses, print exposure was a within-items variable and BOI was a

Table 1 | Mean characteristics for word stimuli.

\begin{tabular}{|c|c|c|c|c|c|c|c|c|c|c|c|c|c|c|}
\hline Word type & BOI & Plen & OFreq & SFreq & $\mathbf{N}$ & PN & PFI & CD & SemD & NumF & Nums & NumA & Conc & Image \\
\hline Low BOI & 3.3 & 4.4 & 17.9 & 3.6 & 6.4 & 13.3 & 3.1 & 0.7 & 307.3 & 3.6 & 4.7 & 13.7 & 5.8 & 6.3 \\
\hline
\end{tabular}

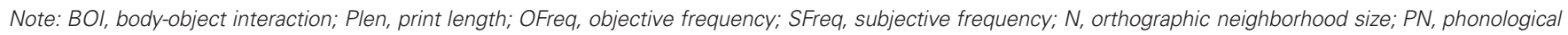

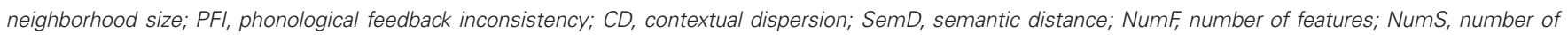
senses; NumA, number of associates; Conc, concreteness; Image, imageability. 
between-items variable. Unless noted, all effects were statistically significant at $p<0.05$. The mean response latencies for correct responses and mean error percentages for the experimental words are presented in Table 2 . The error percentages for the less imageable word foils were $8.5 \%$ for the high print exposure group and $7.3 \%$ for the low print exposure group.

\section{Response latency analysis}

For the response latency data, there was an effect of print exposure, $F_{1}(1,44)=5.05, \mathrm{MSE}=34,743.24, \eta^{2}=0.10 ; F_{2}(1,45)=$ $110.10, \mathrm{MSE}=1593.21, \eta^{2}=0.71$, with the high print exposure group responding on average $88 \mathrm{~ms}$ faster than the low print exposure group. There was an effect of BOI, $F_{1}(1,44)=84.15$, $\mathrm{MSE}=1141.79, \eta^{2}=0.66 ; F_{2}(1,45)=9.14, \mathrm{MSE}=11,532.60$, $\eta^{2}=0.17$, with responses to high BOI words on average $64 \mathrm{~ms}$ faster than responses to low BOI words. There was also an interaction between print exposure and BOI, $F_{1}(1,44)=4.33$, $\mathrm{MSE}=1141.79, \eta^{2}=0.09 ; F_{2}(1,45)=4.96, \mathrm{MSE}=1593.21$, $\eta^{2}=0.10$. This interaction was followed up by analyzing the effects of BOI for each print exposure group separately. For the high print exposure group, the $50 \mathrm{~ms}$ BOI effect was significant, $t_{1}(22)=5.03, \mathrm{SEM}=9.93, \eta^{2}=0.54 ; t_{2}(45)=2.76$, $\mathrm{SEM}=17.62, \eta^{2}=0.15$. For the low print exposure group, the $79 \mathrm{~ms}$ BOI effect was significant, $t_{1}(22)=7.93, \mathrm{SEM}=9.99$, $\eta^{2}=0.74 ; t_{2}(45)=3.00$, SEM $=28.41, \eta^{2}=0.17$. The interaction indicates that although facilitatory effects of BOI were observed for both print exposure groups, the effect was significantly larger for the low print exposure group than for the high print exposure group.

\section{Error analysis}

For the error data, there was no effect of print exposure, both $F$ s $<1$, but there was an effect of BOI, $F_{1}(1,44)=13.27, \mathrm{MSE}=$ $12.64, \eta^{2}=0.23 ; F_{2}(1,45)=4.97, \operatorname{MSE}=32.40, \eta^{2}=0.10$, with responses to high BOI words on average $2.7 \%$ more accurate

Table 2 | Mean raw response latencies (in $\mathrm{ms}$ ) and standard errors, mean error percentages and standard errors, and mean transformed response latencies (in Z-scores) and standard errors for Experiment 1.

\begin{tabular}{|c|c|c|c|c|}
\hline \multirow[t]{2}{*}{ Word type } & \multicolumn{2}{|c|}{ High print exposure } & \multicolumn{2}{|c|}{ Low print exposure } \\
\hline & $M$ & SE & $M$ & SE \\
\hline \multicolumn{5}{|c|}{ RESPONSE LATENCIES } \\
\hline High BOI & 667 & 21.9 & 740 & 30.7 \\
\hline Low BOI & 717 & 24.2 & 819 & 33.4 \\
\hline BOI effect & +50 & & +79 & \\
\hline \multicolumn{5}{|c|}{ RESPONSE ERRORS } \\
\hline High BOI & 0.6 & 0.3 & 1.4 & 0.6 \\
\hline Low BOI & 4.3 & 1.2 & 3.0 & 1.0 \\
\hline BOI effect & +3.7 & & +1.6 & \\
\hline \multicolumn{5}{|c|}{ TRANSFORMED RESPONSE LATENCIES } \\
\hline High BOI & -0.155 & 0.02 & -0.194 & 0.02 \\
\hline Low BOI & 0.170 & 0.03 & 0.202 & 0.02 \\
\hline BOI effect & 0.325 & & 0.396 & \\
\hline
\end{tabular}

Note: BOI, body-object interaction. than responses to low BOI words. There was no interaction between print exposure and BOI, $F_{1}(1,44)=2.03, p=0.16$, $\mathrm{MSE}=12.64 ; F_{2}(1,45)=1.33, p=0.25, \mathrm{MSE}=15.34$.

\section{DISCUSSION}

The purpose of the present experiment was to examine the influence of print exposure on the facilitatory BOI effect in semantic categorization. More specifically, we predicted that there would be a main effect of print exposure (i.e., faster response latencies for the high print exposure readers than for the low print exposure readers) and that the facilitatory BOI effect would be smaller for high print exposure readers than for low print exposure readers. Both of these predictions were borne out by our results.

The above two findings can be accounted for in the following way. Based on the reasoning underlying the lexical integrity hypothesis (Yap et al., 2009), it is likely that the high print exposure readers developed higher integrity semantic representations than did the low print exposure readers. That is, words of a certain objective frequency (in the case of the present study the words were of low frequency) would be closer to semantic recognition threshold for the high print exposure readers due to their more frequent reading experience (to the extent that reading experience is measured by the ART), and would, therefore, result in both faster overall responding, and requiring less simulation of motoric knowledge before responding. The latter outcome would result in less benefit from motor simulation for the high print exposure readers and hence a reduced facilitatory BOI effect. In other words, there is less need for motor simulation to influence the settling of a semantic representation for high print exposure readers.

The above findings (i.e., a main effect of print exposure and a print exposure by BOI interaction) are consistent with the findings of the two studies we noted in our description above of the lexical integrity hypothesis. More specifically, Yap et al. (2009) reported the following two results. First, there was a main effect of group, such that participants from Washington University in St. Louis (WUSTL) had significantly faster overall lexical decision response latencies than did participants from University at Albany, State University of New York (SUNY-A), who also, interestingly, had lower levels of vocabulary knowledge. Second, there was a significant interaction between group, priming, and word frequency, such that the largest priming effect was observed for low-frequency words for the SUNY-A participants. Furthermore, Hargreaves et al. (2012b) reported that non-expert Scrabble players (who had less extensive lexical knowledge) had slower overall lexical decision latencies and larger effects of concreteness than competitive Scrabble players. Thus, our observed findings of a main effect of print exposure and a larger facilitatory effect of BOI for the low print exposure readers are consistent with previous findings in the literature, and with the notion that lexical integrity influences the effects of semantic richness in the semantic categorization task.

Despite the present finding that the facilitatory BOI effect was smaller for the high print exposure readers than for the low print exposure readers, it is worthwhile to emphasize that the effect was still quite large for the former type of reader. This suggests that even though it is likely that the low-frequency words 
used in the present study were associated with higher semantic recognition thresholds for the high print exposure readers, motor simulation still played an important role in lexical conceptual processing for readers with relatively more reading experience in the semantic categorization task, a task in which responses are based on semantic processing. In other words, knowledge gained through sensorimotor experience still exerted a facilitatory effect on lexical conceptual processing for readers who putatively have higher integrity semantic representations. This is an important discovery.

Some researchers, however, might raise an alternative interpretation of our findings (e.g., Faust et al., 1999; Yap et al., $2012 a)^{2}$. For example, Faust et al. stated that "response latencies for different groups are often linearly related, leading to an increased likelihood of finding spurious overadditive interactions in which the slower group produces a larger treatment effect" (p. 777). According to this viewpoint, there are two factors that may contribute to observed group differences for the variable under examination. The first factor is processing rate, which is an individual differences factor, such that slower readers will have on average slower processing rates than faster readers. The second factor is processing amount, which refers to the information processing requirements of the task (e.g., in our Experiment 1, the accumulation of evidence that a word is easily imageable in order to make a "yes" response).

According to this viewpoint, the print exposure by BOI interaction observed in Experiment 1 may be explained in one of two ways. First, it may be that the larger facilitatory BOI effect for the low print exposure readers was primarily due to their receiving greater benefits of motor simulation for the high BOI words, attributable to the processing amount factor discussed above. Second, it may be that the larger facilitatory BOI effect for the low print exposure readers was simply due to their having slower overall response latencies, attributable to the processing rate factor. Needless to say, the second of these explanations is less theoretically interesting, due to the claim that observed group differences are merely due to a correlation with processing rate and not due to differential levels of motor simulation elicited by high BOI and low BOI words for the high print exposure and low print exposure readers.

To differentiate between these two possible explanations, Faust et al. (1999) suggested transforming raw response latencies to a common scale, which would factor out overall group differences in processing rate. By so doing, according to this viewpoint, if the group by variable interaction is still observed after response latency transformation, then it can be attributed to differences in processing amount between the two groups (which would be the theoretically more interesting outcome), but if the group by variable interaction is not observed after the transformation, then the original finding can be simply attributed to processing rate (which would be the theoretically less interesting outcome). Thus, we transformed the raw response latencies using the $z$-score transformation procedure. We then analyzed our $z$-score transformed response latency data using a 2 (print exposure: high, low) $\times 2$ (BOI: high, low) mixed-model ANOVA. Because we believe this is

\footnotetext{
${ }^{2}$ We thank Melvin Yap for bringing this to our attention.
}

a much more conservative test of our data, we employed planned comparisons examining the effect of BOI for each print exposure group separately, regardless of whether the print exposure by BOI interaction was significant. The mean transformed response latencies for correct responses for the experimental words are presented in Table 2 .

\section{Z-score response latency analysis}

There was, of course, no effect of print exposure, $F_{1}(1,44)=$ $1.50, \mathrm{MSE}=0.001 ; \quad F_{2}<1$. There was an effect of BOI, $F_{1}(1,44)=119.77, \mathrm{MSE}=0.025, \eta^{2}=0.73 ; F_{2}(1,45)=8.94$, MSE $=0.22, \eta^{2}=0.17$, with a $z$-score difference between the high BOI words and the low BOI words of 0.292 (and the high BOI words having faster latencies). There was no interaction between print exposure and BOI, both $F \mathrm{~s}<1.40$. For the high print exposure group, the $z$-score difference between the high BOI words and the low BOI words was 0.325 and was significant, $t_{1}(22)=6.42, \operatorname{SEM}=0.05, \eta^{2}=0.65 ; t_{2}(45)=2.76$, $\mathrm{SEM}=0.09, \eta^{2}=0.15$. For the low print exposure group, the $z$-score difference between the high BOI words and the low BOI words was 0.396 and was significant, $t_{1}(22)=9.40, \mathrm{SEM}=0.04$, $\eta^{2}=0.80 ; t_{2}(45)=2.97, \operatorname{SEM}=0.11, \eta^{2}=0.16$. The analysis of the $z$-score transformed response latencies suggests that the original finding of a larger facilitatory effect of BOI for the low print exposure readers was largely due to differences of processing rate between the two print exposure groups, rather than an effect of differential impact of motor simulation elicited by the high BOI words and the low BOI words. We will address this issue in more detail in the General Discussion section.

\section{EXPERIMENT 2}

The experimental design used in Experiment 1 allowed us to examine the effect of print exposure on BOI in a task (semantic categorization) in which responses were based primarily on semantic processing. Recall that according to the semantic feedback framework, semantic processing per se is one of two mechanisms by which semantic richness effects may arise in the visual word recognition system. Further recall that the second such mechanism is feedback from semantics to orthography or to phonology, and thus semantic richness effects can be examined in tasks in which responses are based primarily on either orthographic processing (e.g., lexical decision) or phonological processing (e.g., phonological lexical decision).

As noted, ART scores are associated with various aspects of reading, such as early reading ability, reading experience, and vocabulary knowledge. ART scores are also associated with orthographic and phonological processing differences in skilled readers. For example, Chateau and Jared (2000) used the ART to create groups consisting of either high print exposure readers or low print exposure readers (the two print exposure groups were matched for performance on the comprehension subtest of the Nelson-Denny Reading Test). Chateau and Jared examined orthographic processing using both a homophone choice task, in which participants must choose the correct homophone for a given category (e.g., FRUIT: pear-pair), and a lexical decision task with pseudohomophones as foils; they examined phonological processing using a pseudoword naming task in which participants 
named aloud pseudowords that were not pseudohomophones (e.g., shup); and they jointly examined orthographic and phonological processing in a form priming task (e.g., touch-couch). In each task, the high print exposure readers exhibited more efficient orthographic and/or phonological processing such that: (1) they were significantly faster and more accurate in the homophone choice, lexical decision, and pseudoword naming tasks; (2) they had smaller effects of word frequency and orthographic typicality (i.e., more quickly accepted non-wordlike words and rejected more wordlike pseudohomophones) in the lexical decision task; and (3) they quickly activated orthographic representations when reading high-frequency words, but soon after more strongly activated phonological representations of those words in the form priming task (see Stanovich and West, 1989, for a similar set of results). Chateau and Jared concluded that the increased reading experience of the high print exposure readers led to development of more efficient orthographic and phonological processing skills as compared to the low print exposure readers.

Sears et al. (2008) also used the ART to create groups consisting of either high print exposure readers or low print exposure readers (but unlike Chateau and Jared, 2000, their two print exposure groups were not matched for performance on any other measure). Sears et al. examined the effect of print exposure on the effects of word frequency and neighborhood size (the finding that words with many orthographic neighbors are responded to more quickly than words with few orthographic neighbors; see Siakaluk et al., 2002) in two lexical decision tasks, one in which regular non-words were used and another in which pseudohomophones were used. They reported that high print exposure readers were significantly faster and more accurate, and had significantly smaller effects of word frequency and neighborhood size than did the low print exposure readers only in the lexical decision task in which pseudohomophones were used. In the lexical decision task in which regular non-words were used there were no effects of print exposure. Sears et al. interpreted their findings as indicating that low print exposure readers, "use phonological information to compensate for less efficient orthographic processing skills and this leads to larger word frequency and neighborhood size effects in a lexical decision task when phonology cannot be used to discriminate words from nonwords (such as when pseudohomophones are used in the task)" (p. 289).

Lastly, Unsworth and Pexman (2003) examined phonological processing differences between more skilled readers and less skilled readers (as measured by the ART, and the comprehension and vocabulary subtests of the Nelson-Denny Reading Test) in lexical decision and phonological lexical decision tasks. Their most robust findings were that the more skilled readers responded more quickly and showed no effects of spelling-to-sound regularity in either task, whereas the less skilled readers showed significant effects of spelling-to-sound regularity in both tasks. Unsworth and Pexman concluded that the more skilled readers had developed more efficient phonological processing skills as compared to the less skilled readers. In other words, the more skilled readers had developed more efficient orthographic-tophonological mappings than the less skilled readers.

As noted, a second purpose of the present study was to examine the effects of print exposure on BOI in the phonological lexical decision task, which would allow us to determine whether print exposure influences the effects of BOI on semantic feedback to phonology. As the above review of the literature amply demonstrates, print exposure influences phonological (and orthographic) processing. Experiment 2 provided an opportunity to extend this work by examining whether print exposure influences orthographic-to-semantics-to-phonological mappings, because according to the semantic feedback framework, phonological lexical decision responses are based primarily on phonological processing, but can be modulated by feedback from semantics to phonology.

There are two possible outcomes of the influence of print exposure on BOI in the phonological lexical decision task. The first outcome, according to the lexical integrity hypothesis (Yap et al., 2009), is that a larger effect of BOI should be observed with the low print exposure readers, because their phonological representations should be further away from recognition threshold and would thus benefit more from motor simulation. The second outcome, according to the literature demonstrating that high print exposure readers have more efficient phonological processing, is that a larger effect of BOI may be observed with these readers, because the effects of semantic feedback, derived from motor simulation in semantics, should be more efficiently mapped onto phonological processing for this group of readers.

\section{METHODS}

\section{Participants}

Seventy-two undergraduate students from the University of Northern British Columbia participated in the experiment for bonus course credit. All participants were native English-speakers and reported normal or corrected-to-normal vision. None of these individuals participated in Experiment 1.

Participants were administered the ART after they completed the phonological lexical decision task (described below). For the data analyses, two groups of participants were created by taking the top and bottom $40 \%$ of the ART scores. As such, 29 participants were assigned to the high print exposure group (with a mean ART score of 17.0 and a range of $11-31$ ) and 28 participants were assigned to the low print exposure group (with a mean ART score of 5.6 and a range of $2-9)^{3}$.

\section{Stimuli}

The same set of experimental words used in Experiment 1, and the 48 pseudohomophones and 96 non-words used in the phonological lexical decision task by Siakaluk et al. (2008a) were used in the present experiment. Due to very low error rates, the low BOI item tribe was retained in the present set of analyses. The non-word and pseudohomophone stimuli are listed in the Appendix.

\section{Apparatus and procedure}

The apparatus and procedure were identical to Experiment 1 except for the following. The participants' task was to decide

\footnotetext{
${ }^{3}$ The mean ART score for the low print exposure group in Experiment 2 was higher (and had a larger range) than for the low print exposure group in Experiment 1. This was unavoidable due to the different distributions of ART scores for each group in the two experiments.
} 
whether each letter string sounded like a real English word. Participants were instructed to press the "?" key on the computer keyboard when letter strings that sounded like real English words were presented (i.e., for the experimental words and the pseudohomophones), and to make no response when letter strings that did not sound like real English words were presented (i.e., for the non-words).

Before beginning the experiment, each participant first completed 20 practice trials that consisted of five words similar in normative frequency to the experimental items, five pseudohomophones, and 10 non-words.

\section{RESULTS}

Outliers were identified in the same fashion as in Experiment 1. A total of 102 observations (3.7\% of the data) were removed from the data-set. Only six errors were made (two errors to the high BOI words and four errors to the low BOI words) across all 57 participants, thus no error analysis was conducted.

As was the case for Experiment 1, response latency analyses were conducted on both raw latencies and $z$-score transformed latencies. Each type of latency was analyzed using a 2 (print exposure: high, low) $\times 2$ (BOI: high, low) mixed-model ANOVA, with both subject $\left(F_{1}\right)$ and item $\left(F_{2}\right)$ analyses conducted. The mean raw and $z$-score transformed response latencies for correct responses for the experimental words are presented in Table 3. The mean response latencies for correct responses for the pseudohomophones were $874 \mathrm{~ms}$ for the high print exposure group and $974 \mathrm{~ms}$ for the low print exposure group. The pseudohomophone and non-word error percentages for the high print exposure group were $3.9 \%$ and $8.2 \%$, respectively; for the low print exposure group they were $4.4 \%$ and $10.2 \%$, respectively.

\section{Raw response latency analysis}

For the raw response latency data, there was an effect of print exposure, $F_{1}(1,55)=5.28, \mathrm{MSE}=12,221.37, \eta^{2}=0.09$; $F_{2}(1,46)=111.57, \mathrm{MSE}=521.38, \eta^{2}=0.71$, with the high print exposure group responding on average $47 \mathrm{~ms}$ faster than the low print exposure group. There was an effect of BOI, $F_{1}(1,55)=6.09, \operatorname{MSE}=838.67, \eta^{2}=0.10 ; F_{2}(1,46)=3.43$,

Table 3 | Mean raw response latencies (in $\mathrm{ms}$ ) and standard errors, mean error percentages and standard errors, and mean transformed response latencies (in Z-scores) and standard errors for Experiment 2.

\begin{tabular}{|c|c|c|c|c|}
\hline \multirow[t]{2}{*}{ Word type } & \multicolumn{2}{|c|}{ High print exposure } & \multicolumn{2}{|c|}{ Low print exposure } \\
\hline & $M$ & SE & M & SE \\
\hline \multicolumn{5}{|c|}{ RAW RESPONSE LATENCIES } \\
\hline High BOI & 605 & 14.5 & 660 & 14.5 \\
\hline Low BOI & 626 & 16.3 & 666 & 15.1 \\
\hline BOI effect & +21 & & +6 & \\
\hline \multicolumn{5}{|c|}{ TRANSFORMED RESPONSE LATENCIES } \\
\hline High BOI & -0.083 & 0.03 & -0.014 & 0.03 \\
\hline Low BOI & 0.082 & 0.03 & 0.013 & 0.03 \\
\hline BOI effect & 0.165 & & 0.027 & \\
\hline
\end{tabular}

Note: BOI, body-object interaction. $p=0.07, \mathrm{MSE}=1326.45, \eta^{2}=0.07$, with responses to high BOI words on average $13 \mathrm{~ms}$ faster than responses to low BOI words. There was no interaction between print exposure and BOI, $F_{1}(1,55)=1.76, p=0.19, \mathrm{MSE}=838.67 ; F_{2}(1,46)=$ 2.27, $p=0.14, \mathrm{MSE}=521.38$. Planned comparisons were conducted to examine the effects of BOI for each print exposure group separately. For the high print exposure group, the $21 \mathrm{~ms}$ BOI effect was significant, $t_{1}(28)=2.69, \mathrm{SEM}=7.64, \eta^{2}=0.21$; $t_{2}(46)=2.58, \mathrm{SEM}=8.07, \eta^{2}=0.13$. For the low print exposure group, the $6 \mathrm{~ms}$ BOI effect was not significant, both $t \mathrm{~s}<1$.

\section{Z-score transformed response latency analysis}

For the $z$-score transformed response latency data, there was, of course, no effect of print exposure, both $F$ s $<1$. There was an effect of BOI, $F_{1}(1,55)=4.63, \mathrm{MSE}=0.06, \eta^{2}=0.08$; $F_{2}(1,46)=3.58, p=0.07, \mathrm{MSE}=0.06, \eta^{2}=0.07$, with a $z$-score difference between the high BOI words and the low BOI words of 0.096 (and the high BOI words having faster latencies). There was no interaction between print exposure and BOI, $F_{1}(1,55)=2.42, p=0.13, \operatorname{MSE}=0.06 ; F_{2}(1,46)=2.72$, $p=0.11, \mathrm{MSE}=0.03$. Planned comparisons were conducted to examine the effects of BOI for each print exposure group separately. For the high print exposure group, the $z$-score difference between the high BOI words and the low BOI words was 0.165 and was significant, $t_{1}(28)=2.47, \mathrm{SEM}=0.07, \eta^{2}=0.18$; $t_{2}(46)=2.58, \mathrm{SEM}=0.06, \eta^{2}=0.13$. For the low print exposure group, the $z$-score difference between the high BOI words and the low BOI words was 0.027 and was not significant, both $t$ s $<1$.

\section{Cross task analysis}

In order to statistically evaluate the opposite patterns observed across our two tasks, we also analyzed the data from both tasks together, in a 2 (print exposure: high, low) $\times 2$ (BOI: high, low) $\times$ 2 (task: SCT, PLDT) mixed-model ANOVA. Both subject $\left(F_{1}\right)$ and item $\left(F_{2}\right)$ analyses were conducted. In the subject analyses, print exposure and task were between-subjects variables and BOI was a within-subjects variable. In the item analyses, print exposure and task were within-items variables and BOI was a betweenitems variable. We report only whether the three-way interaction between print exposure, BOI, and task was significant.

For the raw response latency analysis, the three-way interaction was significant, $F_{1}(1,99)=6.24, \mathrm{MSE}=973.39, \eta^{2}=0.06$; $F_{2}(1,91)=7.26, \mathrm{MSE}=1051.41, \eta^{2}=0.07$. For the $z$-score transformed response latency analysis, the three-way interaction approached significance, $F_{1}(1,99)=3.28, p=0.07$, MSE $=$ $0.04, \eta^{2}=0.03 ; F_{2}(1,91)=3.95, p=0.05, \operatorname{MSE}=0.02, \eta^{2}=$ 0.04 . These analyses confirm that there were significantly different interactions of print exposure and BOI in our two experiments: whereas high print exposure readers were faster than low print exposure readers in both tasks, they showed smaller BOI effects (than low print exposure readers) in the semantic categorization task and larger BOI effects (than low print exposure readers) in the phonological lexical decision task.

\section{DISCUSSION}

Recall that two outcomes were proposed regarding the possible influence of print exposure on BOI in the phonological lexical 
decision task. The first proposed outcome was that a larger BOI effect should be observed with the low print exposure readers, because their lower integrity phonological representations should be further away from recognition threshold and would, therefore, benefit more from the greater motor simulation elicited by the high BOI words. The second proposed outcome was that a larger effect of BOI may be observed with the high print exposure readers, because they have more efficient orthographicto-semantics-to-phonology mappings that would allow them to benefit more from the greater motor simulation elicited by the high BOI words.

The two key results from Experiment 2-that the high print exposure readers responded more quickly and were the only print exposure group to have a facilitatory BOI effect-clearly do not support the first proposed outcome, but instead provide support for the second proposed outcome. That is, these two findings are consistent with the idea that print exposure develops more efficient feedback from semantics to phonology, with the end result of faster settling of phonological representations associated with high BOI words than of phonological representations associated with low BOI words, thus producing faster phonological lexical decision latencies for the former type of words. Furthermore, the findings from the present experiment extend previous work examining the effects of print exposure on orthographic and phonological processing, by suggesting that print exposure also develops more efficient orthographic-to-semanticsto-phonological mappings within the visual word recognition system.

\section{GENERAL DISCUSSION}

In the present study we examined the influence of print exposure on BOI in visual word recognition, using the semantic categorization and phonological lexical decision tasks. Our findings suggest that print exposure does influence the BOI effect, and that the manner by which it does so depends on task demands.

Recall that according to the semantic feedback framework, responses in the semantic categorization task are primarily based on semantic processing, and that a facilitatory effect of BOI arises under these conditions because high BOI words elicit richer semantic activation (i.e., richer motor simulations) within the semantic units, which leads to faster settling of semantic representations and faster semantic categorization latencies for these types of words. The results from our raw latency analyses indicated that although there was a facilitatory BOI effect for both print exposure groups, the effect was significantly smaller for the high print exposure group. An extension of the lexical integrity hypothesis (Yap et al., 2009) provides an explanation for why this finding was observed. More specifically, for high print exposure readers, greater reading experience leads to the development of higher integrity semantic representations that are closer to recognition threshold (or, in other words, settle more quickly), and to greater efficiency of semantic processing more generally. This results in less benefit for these readers when motor simulations are used to make semantic categorizations, because less stimulus-driven knowledge is needed to correctly categorize the stimuli, which leads to less of an advantage in the recognition of and responding to high BOI words. Conversely, for low print exposure readers, less reading experience leads to the development of lower integrity semantic representations that are further away from recognition threshold (or, in other words, settle less quickly), and to less efficient semantic processing more generally. This results in more benefit for these readers when motor simulations are used to make semantic categorizations, because more stimulus-driven knowledge is needed to correctly categorize the stimuli, which leads to more of an advantage in the recognition of and responding to high BOI words. In summary, under experimental conditions in which responses are primarily based directly on semantic processing, higher integrity representations and more efficient processing leads to an attenuation of the facilitatory BOI effect.

We noted in the Discussion section of Experiment 1 that there is an alternative viewpoint regarding the interpretation of the print exposure by BOI interaction observed in our semantic categorization results. According to this viewpoint (Faust et al., 1999; Yap et al., 2012a), it is difficult to determine, using raw response latencies, whether the larger facilitatory BOI effect associated with the low print exposure group was due to a greater impact of motor simulation elicited by the high BOI words than the low BOI words for this group of readers (i.e., it is due to processing amount), or if the BOI effect was simply correlated with individual differences in overall response latencies (i.e., it is due to processing speed or, in other words, the low print exposure readers had a larger facilitatory BOI effect simply because they took longer to respond). In order to disentangle these two possibilities, Faust et al. suggested transforming raw response latencies to a common scale, in essence statistically partialling out any effect attributable to processing rate, and only examining any effect that may be attributable to processing amount. If the print exposure by BOI interaction remained after such a transformation of the response latency data, then, according to this viewpoint, the interpretation of differential benefits of motor simulation between the two print exposure groups is valid; otherwise, no such interpretation is warranted. Recall that after $z$-score transforming our raw response latency data, there was no longer a print exposure by BOI interaction. In other words, the facilitatory BOI effect could be interpreted as being of similar magnitude for both print exposure groups.

The analyses based on the raw semantic categorization latencies and the $z$-score transformation of those latencies lead to different conclusions. The raw response latency findings support the idea that motor simulation is of differential benefit for low print exposure readers and high print exposure readers, whereas the $z$-score transformed response latency findings support the idea that there is little, if any, differential benefit of motor simulation between the two print exposure groups. We propose that although there may be instances in which the alternative viewpoint of group by variable interactions as indicative of "spurious overadditive interactions" (Faust et al., 1999, p. 777) is valid there may be instances in which this is an overly restrictive way of interpreting these types of interactions. For example, if there is reason to suspect that the groups under examination may have qualitative differences in how they process information (such as comparing older participants vs. younger participants, or brain-injured vs. non-brain-injured participants, on some cognitive task), it is likely appropriate to consider processing rate 
as a variable having two relatively distinct kinds of processing. In these types of cases, partialling out the effects of processing rate would be warranted before interpreting a group by variable interaction. However, if there is reason to suspect that the groups under examination may simply have quantitative differences in how they process information (such as comparing two groups of university-based, skilled readers), it is likely inappropriate to consider processing rate as a variable having two relatively distinct kinds of processing. In these types of cases, which we suggest is indicative of the present study, partialling out the effects of processing rate would not be necessary to interpret a group by variable interaction. Thus, we conclude that print exposure did differentially influence the effects of BOI for the high print exposure readers and low print exposure readers in Experiment 1, although we acknowledge that some researchers may disagree with this interpretation.

Recall that according to the semantic feedback framework, responses in the phonological lexical decision task are primarily based on phonological processing, and that a facilitatory BOI effect arises in this task because high BOI words elicit greater semantic activation (i.e., richer motor simulations) within the semantic units, which then sends stronger semantic feedback to the phonological units, leading to faster settling on phonological representations and hence faster phonological lexical decision latencies. The results from Experiment 2 indicated that a facilitatory BOI effect was observed only for the high print exposure readers. These results are consistent with the idea that reading experience leads to the development of more efficient orthographic-to-phonological-to-semantic mappings, a novel finding, and when this occurs, the effects of semantic feedback are more beneficial for the high print exposure readers, readers whose visual word recognition processes benefit from the increased semantic feedback to phonology that is elicited by high BOI words. Importantly, these conclusions are immune from the criticism of the alternative viewpoint proposed by Faust et al. (1999), because the high print exposure readers exhibited both faster overall phonological lexical decision latencies, and were the only print exposure group for which a facilitatory BOI effect was observed.

In summary, under experimental conditions in which responses are not based directly on semantic processing, but

\section{REFERENCES}

Andrews, S., and Bond, R. (2009). Lexical expertise and reading skill: bottom-up and top-down processing of lexical ambiguity. Read. Writ. 22, 687-711.

Andrews, S., and Hersch, J. (2010). Lexical precision in skilled readers: individual differences in masked neighbor priming. J. Exp. Psychol. Gen. 139, 299-318.

Andrews, S., and Lo, S. (2012). Not all skilled readers have cracked the code: individual differences in masked form priming. J. Exp. Psychol. Learn. Mem. Cogn. 38, 152-163.

rather may be modulated by semantic feedback, higher integrity representations (i.e., in the case of our Experiment 2, phonological representations) and more efficient processing (i.e., orthographic-to-semantics-to-phonological processing) leads to an increase of the facilitatory BOI effect.

An important limitation of the present study is the reading measure used. Although ART scores are reliably associated with reading experience (Cunningham and Stanovich, 1990) and vocabulary knowledge (West et al., 1993; Lee et al., 1997), they do not capture either dimension perfectly, and there are more precise ways of measuring lexical expertise. For example, Andrews and colleagues have derived and used individual difference measures in written language proficiency with tests of reading, spelling, and vocabulary, and examined the effects of those differences on a number of aspects of lexical retrieval (e.g., masked neighbor priming, Andrews and Hersch, 2010; masked form priming, Andrews and Lo, 2012). This multidimensional approach to assessing lexical expertise should be adopted in future studies of individual differences and semantic processing.

In conclusion, the results of the present study are consistent with the view that lexical conceptual processing is flexible and dynamic in nature (Kiefer and Pulvermüller, 2012). In order to optimize performance in a task, participants can modulate the processing required. More specifically, as a function of print exposure, there is variance in the degree to which embodied semantic information influences lexical conceptual processing. The effects of the semantic richness variable BOI are smaller for high print exposure readers compared to low print exposure readers under conditions in which responses are based on semantic processing (and the former type of readers have higher integrity semantic representations and more efficient semantic processing), but are larger under conditions in which responses are based on phonological processing but may be modulated by semantic feedback (they have higher integrity phonological representations and more efficient orthographic-to-semantics-to-phonological processing).

\section{ACKNOWLEDGMENTS}

This research was supported by Natural Sciences and Engineering Research Council of Canada (NSERC) discovery grants to Paul D. Siakaluk and Penny M. Pexman.

599 multisyllabic nouns. Behav. Res. Methods 43, 1100-1109.

Chateau, D., and Jared, D. (2000). Exposure to print and word recognition processes. Mem. Cogn. 28, $143-153$.

Clark, A. (1997). Being There: Putting Brain, Body, and World Together Again. Cambridge, MA: MIT Press. Cunningham, A. E., and Stanovich, K E. (1990). Assessing print exposure and orthographic processing skill in children: a quick measure of reading experience. J. Educ. Psychol. 82, 733-740.

Cunningham, A. E., and Stanovich, K. E. (1997). Early reading acquisition and its relation to reading experience and ability 10 years later. Dev. Psychol. 33, 934-945.

Faust, M. E., Balota, D. A., Spieler, D. H., and Ferraro, F. R. (1999). Individual differences in information-processing rate and amount: implications for group differences in response latency. Psychol. Bull. 125, 777-799.

Grèzes, J., and Decety, J. (2001). Functional anatomy of execution mental simulation, observation, and verb generation of actions: a meta-analysis. Hum. Brain Mapp. 12, 1-19. 
Hargreaves, I. S., Leonard, G. A., Pexman, P. M., Pittman, D. J., Siakaluk, P. D., and Goodyear, B. G. (2012a). The neural correlates of the body-object interaction effect in semantic processing. Front. Hum. Neurosci. 6:22. doi: 10.3389/fnhum. 2012.00022

Hargreaves, I. S., Pexman, P. M., Zdrazilova, L., and Sargious, P. (2012b). How a hobby can shape cognition: visual word recognition in competitive Scrabble players. Mem. Cogn. 40, 1-7.

Hino, Y., and Lupker, S. J. (1996). Effects of polysemy in lexical decision and naming: an alternative to lexical access accounts. J. Exp. Psychol. Hum. Percept. Perform. 22, 1331-1356.

Hino, Y., Lupker, S. J., and Pexman, P. M. (2002). Ambiguity and synonymy effects in lexical decision, naming, and semantic categorization tasks: interactions between orthography, phonology, and semantics. J. Exp. Psychol. Learn. Mem. Cogn. 28, 686-713.

ITP Nelson Canadian Dictionary. (1997). ITP Nelson Canadian Dictionary of the English Language: An Encyclopedic Reference. Scarborough, ON: ITP Nelson.

Kiefer, M. and Pulvermüller, F. (2012). Conceptual representations in mind and brain: theoretical developments, current evidence and future directions. Cortex. [Epub ahead of print].

Lakoff, G., and Johnson, M. (1999). Philosophy in the Flesh: The Embodied Mind and its Challenge to Western Thought. New York, NY: Basic Books.

Lee, S-Y., Krashen, S., and Tse, L. (1997). The author recognition test and vocabulary knowledge: a replication. Percept. Mot. Skills 85, 1428-1430.

Pecher, D. (2001). Perception is a twoway junction: feedback semantics in word recognition. Psychon. Bull. Rev. 8, 545-551.

Pecher, D., and Zwaan, R. A. (2005). "Introduction to grounding cognition," in Grounding Cognition:
The Role of Perception and Action in Memory, Language, and Thinking, eds D. Pecher and R. A. Zwaan, (Cambridge, UK: Cambridge University Press), 1-7.

Péran, P., Démonet, J. F., Cherubini, A., Carbebat, D., Caltagirone, C. and Sabatini, U. (2010). Mental representations of action: the neural correlates of the verbal and motor components. Brain Res. 1328, 89-103.

Perfetti, C. A. (1992). “The representation problem in reading acquisition," in Reading Acquisition, eds P. B. Gough, L. C. Ehri, and R. Treiman (Hillsdale, NJ: Erlbaum), 145-174.

Perfetti, C. A., and Hart, L. (2002). "The lexical quality hypothesis," in Precursors of Functional Literacy, eds L. Vehoeven, C. Elbro, and P. Reitsma (Amsterdam: John Benjamins), 180-213.

Pexman, P. M., and Lupker, S. J. (1999). Ambiguity and visual word recognition: can feedback explain both homophone and polysemy effects? Can. J. Exp. Psychol. 53, 323-334.

Sears, C. R., Siakaluk, P. D., Chow, V. C., and Buchanan, L. (2008). Is there an effect of print exposure on the word frequency effect and the neighborhood size effect? J. Psycholinguist. Res. 37, 269-291.

Siakaluk, P. D., Buchanan, L., and Westbury, C. (2003). The effect of semantic distance in yes/no and go/no-go semantic categorization tasks. Mem. Cogn. 31, 100-113.

Siakaluk, P. D., Pexman, P. M., Aguilera, L., Owen, W. J., and Sears, C. R. (2008a). Evidence for the activation of sensorimotor information during visual word recognition: the bodyobject interaction effect. Cognition 106, 433-443.

Siakaluk, P. D., Pexman, P. M. Dalrymple, H-A. R., Stearns, J., and Owen, W. J. (2011). Some insults are more difficult to ignore: the embodied insult Stroop effect. Lang. Cogn. Process. 26, 1266-1294.
Siakaluk, P. D., Pexman, P. M., Sears, C. R., and Owen, W. J. (2007). Multiple meanings are not necessarily a disadvantage in semantic processing: evidence from homophone effects in semantic categorisation. Lang. Cogn. Process. 22, 453-467.

Siakaluk, P. D., Pexman, P. M., Sears, C. R., Wilson, K., Locheed, K., and Owen, W. J. (2008b). The benefits of sensorimotor knowledge: body-object interaction facilitates semantic processing. Cogn. Sci. 32 591-605.

Siakaluk, P. D., Sears, C. R., and Lupker, S. J. (2002). Orthographic neighborhood effects in lexical decision: the effects of nonword orthographic neighborhood size. J. Exp. Psychol. Hum. Percept. Perform. 28, 661-681.

Stanovich, K. E., and West, R. F. (1989). Exposure to print and orthographic processing. Read. Res. Q. 24, 402-433.

Tillotson, S. M., Siakaluk, P. D., and Pexman, P. M. (2008). Body-object interaction ratings for 1,618 monosyllabic nouns. Behav. Res. Methods 40, 1075-1078.

Tousignant, C., and Pexman, P. M. (2012). Flexible recruitment of semantic richness: context modulates body-object interaction effects in lexical-semantic processing. Front. Hum. Neurosci. 6:53. doi: 10.3389/fnhum.2012.00053

Unsworth, S. J., and Pexman, P. M (2003). The impact of reader skil on phonological processing in visua word recognition. Q. J. Exp. Psychol. 56, 63-81.

Wellsby, M., Siakaluk, P. D., Owen, W. J., and Pexman, P. M. (2011) Embodied semantic processing: the body-object interaction effect in a non-manual task. Lang. Cogn. $3,1-14$.

West, R. F., Stanovich, K. E., and Mitchell, H. R. (1993). Reading in the real world and its correlates. Read. Res. Q. 28, 34-50.

Wilson, M. (2002). Six views of embodied cognition. Psychon. Bull. Rev. 9 625-636.
Yap, M. J., Balota, D. A., Sibley, D. E., and Ratcliff, R. (2012a). Individual differences in visual word recognition: insights from the English Lexicon Project. J. Exp. Psychol. Hum. Percept. Perform. 38, 53-79.

Yap, M. J., Pexman, P. M., Wellsby, M., Hargreaves, I. S., and Huff, M. J. (2012b). An abundance of riches: cross-task comparisons of semantic richness effects in visual word recognition. Front. Hum. Neurosci. 6:72. doi: 10.3389/fnhum. 2012.00072

Yap, M. J., Tse, C-S., and Balota, D. A. (2009). Individual differences in the joint effects of semantic priming and word frequency revealed by RT distributional analyses: the role of lexical integrity. J. Mem. Lang. 61, 303-325.

Zwaan, R. A. (2008). "Experiential traces and mental simulations in language comprehension," in Symbols and Embodiment: Debates on Meaning and Cognition, eds $\mathrm{M}$. de Vega, A. M. Glenberg, and A. C. Graesser, (New York, NY: Oxford University Press), 165-180.

Conflict of Interest Statement: The authors declare that the research was conducted in the absence of any commercial or financial relationships that could be construed as a potential conflict of interest.

Received: 18 January 2012; accepted: 13 April 2012; published online: 03 May 2012.

Citation: Hansen D, Siakaluk PD and Pexman PM (2012) The influence of print exposure on the body-object interaction effect in visual word recognition. Front. Hum. Neurosci. 6:113. doi: 10.3389/fnhum.2012.00113

Copyright (c) 2012 Hansen, Siakaluk and Pexman. This is an open-acces article distributed under the terms of the Creative Commons Attribution Non Commercial License, which permits non-commercial use, distribution, and reproduction in other forums, provided the original authors and source are credited. 


\section{APPENDIX \\ ITEMS USED IN THE EXPERIMENTS \\ High BOI words}

belt, brick, couch, crown, crumb, dish, drum, fence, flute, gift, grape, lamp, mask, pear, pipe, purse, rope, skirt, stool, suit, tape, thorn, tool, vest

\section{Low BOI words}

cake, cliff, cloud, clown, creek, dirt, ditch, dorm, flame, flood, juice, kite, lace, leaf, mist, pond, seed, shelf, ship, silk, smog, torch, tribe, tube

\section{Less imageable words}

chasm, clout, cusp, farce, fare, fate, fault, feat, flaw, fleck, fluke, fraud, froth, gist, hint, hoax, lack, lapse, loss, luck, noun, oath, pact, pang, phase, plea, ploy, pride, proof, prose, realm, risk, sake, scorn, sect, skill, soul, span, spoof, tact, trait, trend, truce, trust, verb, whiff, whim, zeal

\section{Pseudohomophones}

berd, boal, boan, bote, brane, crain, dait, doar, drane, gaim, gard, goast, gote, groop, gurl, hoam, hoap, hoze, jale, jerm, jirk, joak, klaim, koast, nale, noat, nurve, rane, rong, rore, roze, scail, sheat, shurt, skalp, skarf, sleap, smoak, stawl, stoar, swet, teath, thret, tode, treet, tutch, werk, wheet

\section{Non-words}

bame, beal, besh, bime, binch, bope, bram, brame, brank, brate, bulch, chate, cheen, clace, clirp, crong, cruss, dack, dake, dawk, dreeb, dunch, duss, fage, filt, fitch, flane, flang, flef, flet, foom, fulk, fung, gake, gick, glank, gless, grabe, grafe, gurse, hain, hape, hean, helt, hife, hine, jick, jote, kine, kooce, loke, ludge, meep, merch, moach, nent, nerbe, pake, pame, pape, pell, petch, pilk, pleap, poote, potch, pribe, prog, pung, rame, rask, rell, scaff, scug, shate, shink, slirt, soat, spale, spen, spoop, stort, strup, tain, talt, tane, tark, thurn, tinch, toin, trake, treen, trine, turt, vank, yelf 